\title{
Data Fusion in Application of Image Information
}

\author{
M. PISZCZEK*
}

Institute of Optoelectronics, Military University of Technology, S. Kaliskiego 2, 00-908 Warsaw, Poland

This paper presents a new look at meaning of metadata in applications using image information. Information potential of images is closely associated with the access to such data as internal and external orientation. It is also essential that advanced vision systems are characterized by individual sets of metadata like e.g. range-gated imaging technology. Linking data mentioned above offers synergetic effects. The camera range-gated imaging is getting functionalities characteristic of the radar and scanner 3D. Technology of augmented reality in connecting with the database offers the functionality of the autonomous guide to real world. Possible functionalities are of course a lot and they depend on the equipment configuration of applied sensor sets. Expressed deliberations about data fusion in vision information systems are based on research activity. Expressed issues concerning modeling, equipment configurations and model results of examinations, point on the great information potential available in advanced vision systems.

PACS: $42.30 . \mathrm{Sy}, 42.30 . \mathrm{Tz}, 02.60 .-\mathrm{x}$

\section{Introduction}

Data fusion is generally defined as the use of techniques that combine data from multiple sources. Information obtained in this way will be more efficient and potentially more accurate than if they were achieved by means of a single source. That kind of technique is very useful in the information technology. In the geospatial domain, data fusion is often synonymous with data integration. The solutions in range of information technologies of real time begin playing very essential part. The access to information is unusually essential with point of view of the functioning the systems of public safety. Spatial information and particularly the computer imaging on background of digital map is more and more universally the applied method of integration of information delivered from different sources. Dynamically the developing sensor technique is disclosing valuable data for data integration and data fusion. The development of image science and technology as well as new teleinformatic systems makes possible the study of new solutions for distributed systems. The use of image metadates in connection with the classic observation systems, the panoramic image systems and the space-time photoframe technology contributes to the essential growth of the information potential of terrophotogrametrics studies.

\section{Theoretical background}

What is image information? How can we manage information of this type? Image information is both an image and data describing the image as well as results of the fusion of this data. Typical metadata is e.g. a resolution of the image, moment of acquisition etc. It turns out that along with the rise in the amount of descriptive data an information potential of the image is also growing. The

\footnotetext{
* e-mail: mpiszczek@wat.edu.pl
}

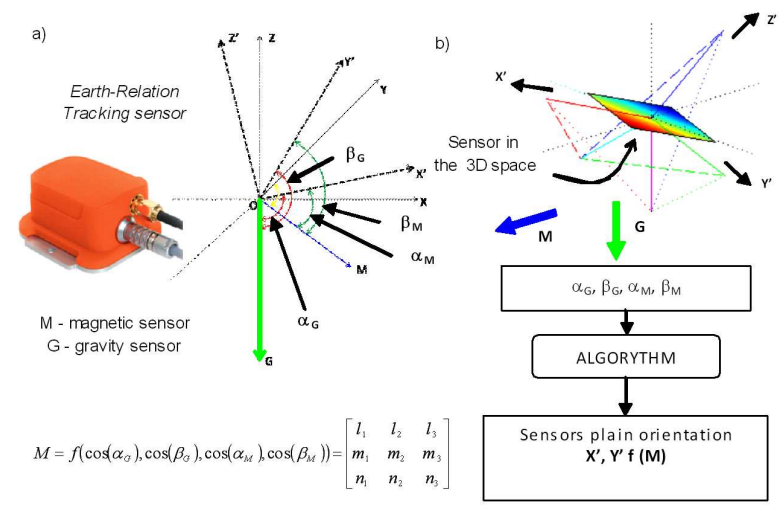

Fig. 1. (a) The module and its orientation in the 3D space, (b) algorithm of obtaining metadata.

time and the location are sufficient for the identification of events. Elements of internal and external orientation enable conducting photogrametric analyses (Fig. 1).

The characteristic parameters of the method of the space-temporal framing (Fig. 2) enable three-dimensional modeling the observed scene.

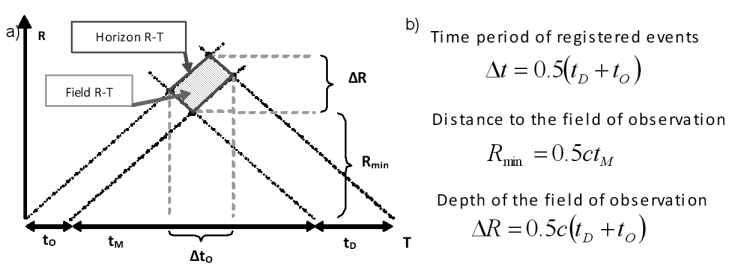

Fig. 2. (a) The spatio-temporal framing, (b) characteristic parameters of the method.

Many ways of linking data and images exist. Interesting examples of solutions using metadata are technologies: range-gated imaging (RGI) and augmented reality $(\mathrm{AR})$. 
The RGI technology uses the method of spatio-temporal framing [1-9]. The method is based on sending short lighting impulses and the synchronous recording of images. Examples of data integration in the RGI technology and connected monitoring-measuring functionalities:

a) for series of images there are getting: differencing and cumulative analysis, improvement in the image contrast thanks to radiometric and range analysis,

b) for images and metadata associated with the spatio-temporal framing (M_STF): spatial selection of objects, evaluation of the distance and oblong dimensions of the object,

c) for images, M_STF and metadata associated with the elements of internal orientation (M_IO): dimensioning of objects,

d) for images, M STF and metadata associated with the elements of external orientation (M_EO): spatial location of objects,

e) for images, M STF, M EO and metadata associated with the elements of internal orientation (M IO): three-dimensional modeling objects and area.

From information point of view particularly a role of the man in the system is interesting. Antropotechnical structure man-machine starts playing a significant role in IT systems. Man becomes fully functional information unit thanks appropriate equipment (for example: Land Warrior). In solutions of this type the AR technology is being used.

AR technology is special solution for a live direct or an indirect view of a real-world environment whose elements are augmented by computer-generated sensory input, such as sound or graphics [9-11]. Resulting functionalities of joining images and data into the AR:

a) for images and metadata associated with the elements of internal and external orientation (M IO, M EO): the sector and direction of observation,

b) for above data and the database of objects in surroundings: identification of objects in the space, distance determination to objects.

\subsection{Possibilities of suggested solutions applications}

Having right information is a base of functioning of people as well as technical systems created by them. Ordered set of data according to needs of the user is a condition of taking by him appropriate action. In the general perspective the imaging system of spatial information (ISSI) is a distributed territorially network of providers and recipients of information. The conception of the object-oriented approach to image information makes easier the implementation of solutions for ISSI. Providers of information can be: people, stationary and mobile sensor modules, decision unit equipped or having an access to different kind of databases. Recipients of information (different degree of processing) can be: anthropotechnical systems equipped with the essential communications and visualisation modules, centres of the monitoring as well as autonomous systems. Proposed concept of functioning of ISSI is to carry integration out through teleinformatic systems into the connected information system.
Real possibilities of the any vision information system results from the competent data processing coming from different territorially distributed sources. Among others a possibility of determining the shape, the size, or spatial location of objects is deciding on the information potential of photogrametric solutions.

Visional systems operating on metadata can perform monitoring-measuring tasks:

- tracking objects in spatial coordinates,

- detecting camouflaged objects,

- detecting objects hidden from view,

- detecting objects behind the fog, the smoke, the fire,

- making models 3D.

\section{Experimental arrangement}

\subsection{Virtual research environment}

Important thing in the research on the fusion of data is possibility of modeling the system and its implementation in the target application. Virtual models of real world can be elements of AR databases. Simulations of the propagation of the radiation will enable the algorithmization of controlling the real device.

Simulation researches on monitoring and measuring functionalities were made using different applications done in MATLAB and SIMULINK.

a) Virtual test environment for basic research in integration of data.

This environment offers the possibility of modeling the observed space as well as parameters image and data acquisition.

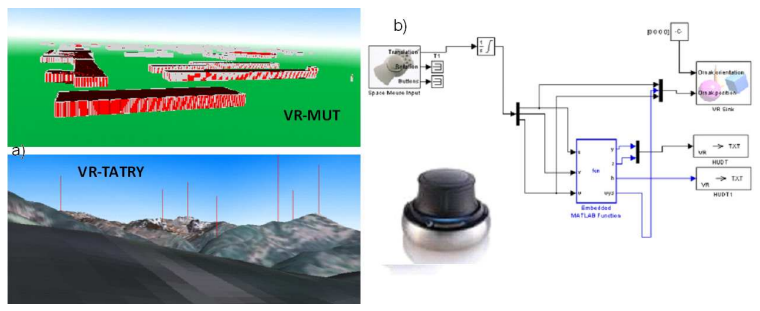

Fig. 3. (a) Virtual models VR-MUT, VR-TATRY, (b) navigation in virtual world.

b) Virtual models of the real area.

Depending on the scale to tests were made (Fig. 3):

- VR-TATRY - model of the Tatra mountains,

- VR-MUT - model of the selected area of the Military University of Technology,

— VR-ROOM - model of the university room. 
For the navigation in virtual models there is used SIMULINK environment with the possibility of the service of manipulator 3D Connexion SpaceNavigator.

c) Environment for modeling RGI technology.

There is a possibility of the synthesis of the classical images as well as the RGI images for the determined distance and the depth of the acquisition (Fig. 4).

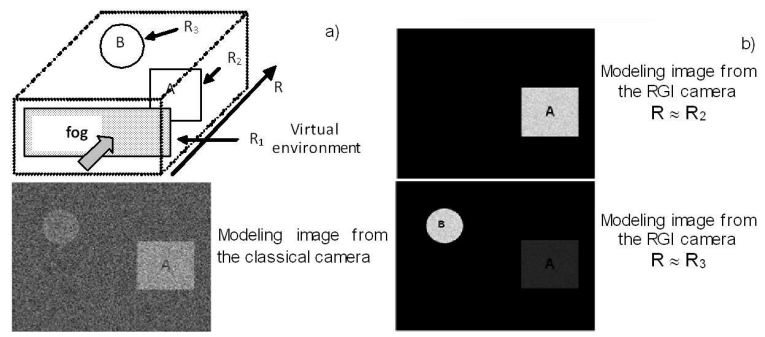

Fig. 4. (a) Virtual environment and classical image, (b) generated images with RGI method for different distances.

\subsection{Test equipment configurations}

Technique of the image acquisition with RGI method requires using three basic components: nanosecond laser illuminator, camera with the amplifier (MCP) and the nanosecond shutter and sequencer synchronizing the functioning of the transmitter and the receiver. RGI functionalities were tested together with the ERT module, the radar and the VIS/IR camera. Main components of the test set and parameters were described in Fig. 5.

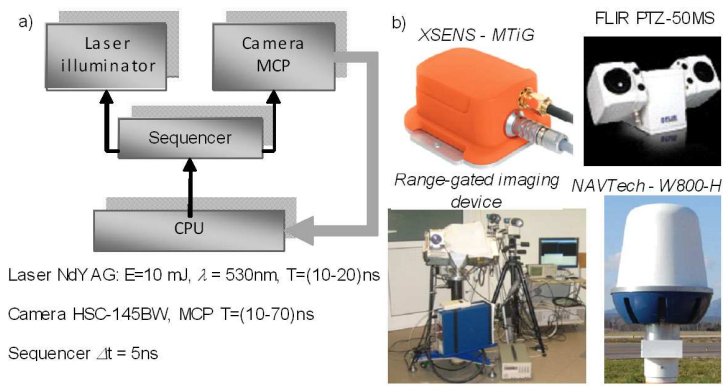

Fig. 5. (a) The scheme and parameters of RGI device, (b) configuration of the system.

The main hardware components for AR are:

- computer - analyzes the sensed visual and other data to synthesize and position augmentations,

- display: head-mounted, handheld and spatial,

- sensors: accelerometers, GPS, gyroscopes, solid state compasses.

Two technologies of linking real and virtual worlds are applied in AR systems: optical (see-through) and video (video-mixing) (Fig. 6). To tests, video-mixing method was used.

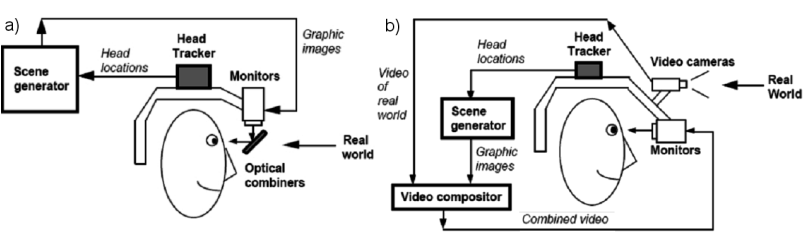

Fig. 6. (a) System HMD see-through, (b) system HMD video-mixing [9].

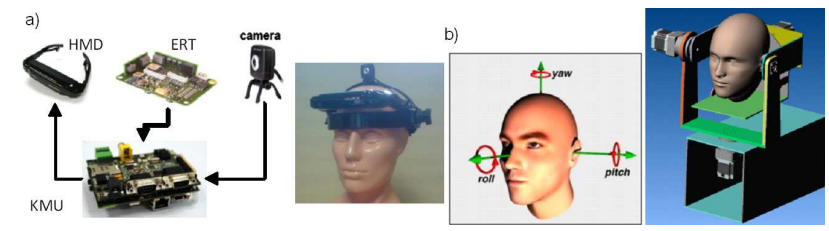

Fig. 7. (a) Equipping the mobile user of the AR system, (b) test platform - 3-axis gimbal set.

In tested solutions the following main components were used (Fig. 7):

- Computer of the Mobile User (KMU) - ARM Cortex A8,

— head-mounted display (HMD) — VR920,

— Eart-Relation Tracking ERT — XSENS-MTi.

Functionalities of AR components were tested on 3 -axis gimbal set.

\section{Results and conclusions}

The results presented below are chosen examples from examinations.

a) The controlled merger of images is one of examples of using metadata in the processing of image information (Fig. 8a). Using metadata increases the speed of the process.

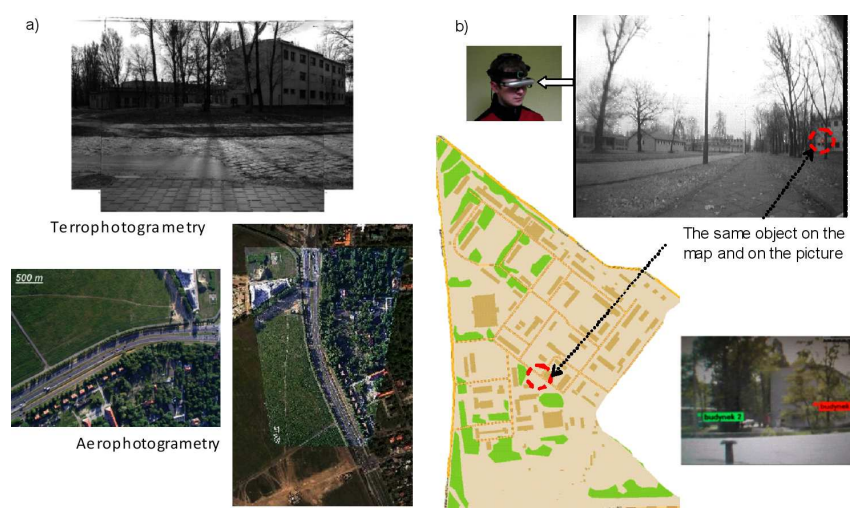

Fig. 8. (a) Image mosaicing, (b) showing the object in a picture. 

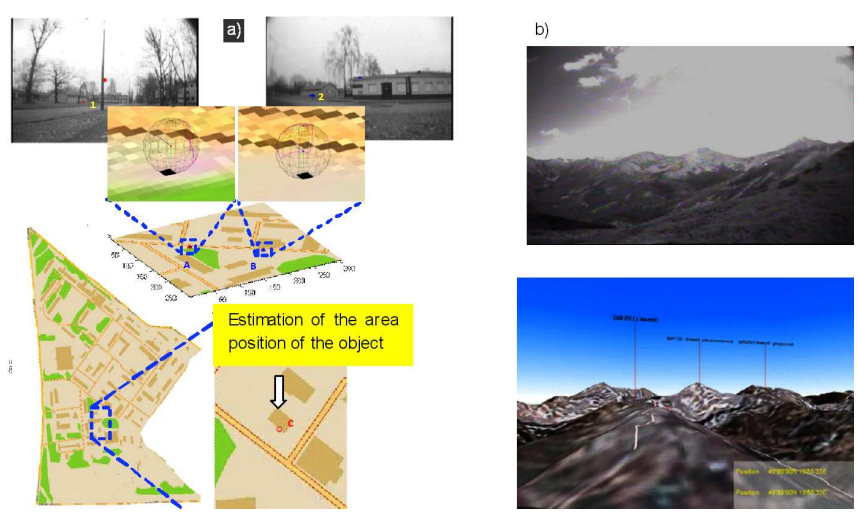

Fig. 9. (a) Positioning of the field point based on analysis of images from two different locations, (b) real and virtual visualisation of the area.
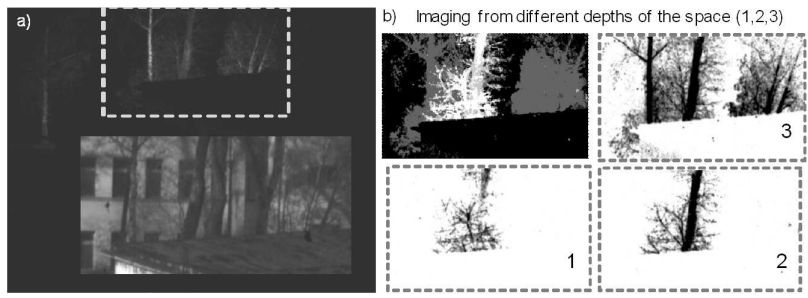

Fig. 10. Selectivity of imaging: (a) classical image, (b) RGI images with different depths.

b) Showing objects on visional material is possible if we know the position of objects in the area and the elements of internal and external orientation cameras (Fig. 8b). The property of this type is one of basic functionalities of AR systems.

c) There is a possible positioning of objects in the space thanks to distributed territorially vision systems (Fig. 9a).

d) The virtual representation of the real image is possible if there are well-known parameters of the image acquisition and database of characteristic objects of surroundings (Fig. 9b).

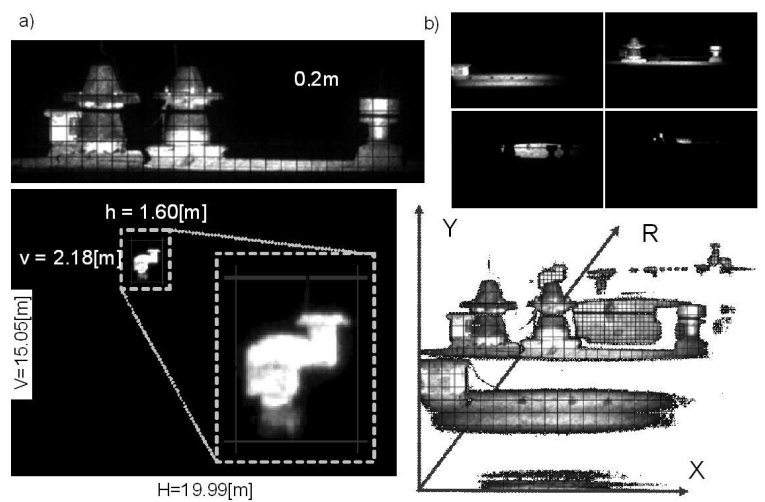

Fig. 11. Dimensioning and modeling objects with RGI method. e) The RGI technology enables more contrasting images. Selective imaging the space is minimizing the influence of atmospheric factors as well as it is increasing in the detestability of objects (Fig. 10).

f) Spatial analyses are one of the most interesting RGI functionalities. Series of photographs with different spatial parameters enable to calculate the geometry of objects as well as modeling the observed scene (Fig. 11).

Presented examples are demonstrating the great potential which is hidden in images and how metadata is important for getting this information.

\section{Acknowledgments}

The expert opinions concern issues of image information was prepared for the Ministry of Defence in 2005. In the same time the work started on issues concerning integration of visual data and other type of data. Survey results are starting appliqué character. The author of the study wants to thank companies: HARDSoft for the cooperation in RGI and SOYTER for the cooperation in AR. The investigations are financed as the grant of Ministry of Science and Higher Education No. OR00000312.

\section{References}

[1] T.E. McDonald, Jr., G.J. Yates, F.H. Cverna, R.A. Gallegos, S.A. Jaramillo, D.M. Numkena, J.R. Payton, Proc. SPIE 3642, 142 (1999).

[2] P. Andersson, Opt. Eng. 45, 034301 (2006).

[3] M. Piszczek, K. Rutyna, M. Szustakowski, in: IX National Conf. COE-2006, 2006, p. 55 (in Polish).

[4] M. Piszczek, K. Rutyna, M. Szustakowski, SPIE Europe Optics and Optoelectron. 65851 L-1, 347 (2007).

[5] M. Piszczek, K. Rutyna, M. Szustakowski, Pomiary Automatyka Kontrola 53, nr 9bis/2007, 531 (2007) (in Polish).

[6] M. Piszczek, K. Rutyna, M. Szustakowski, Europ. Phys. J.-Special Topics 154, 153 (2008).

[7] M. Piszczek, K. Rutyna, Roczniki Geomatyki VI, 73 (2008) (in Polish).

[8] M. Piszczek, K. Rutyna, Elektronika 2/2009, 31, (2009) (in Polish).

[9] M. Piszczek, P. Zagrajek, R. Ryniec, Scientific Notebook of Gdańsk University of Technology - Serie FETI 19/2010, 47 (2010) (in Polish).

[10] R.T. Azuma, Presence: Teleoperators Virtual Environ. 6, 4 (1997).

[11] P. Pardel, Studia Informatica 30, 35 (2009). 